\title{
De Sitter and Power-law Solutions in Non-local Gauss-Bonnet Gravity
}

\author{
E. Elizalde
}

1) Instituto de Ciencias del Espacio (ICE/CSIC)

Campus UAB, Carrer de Can Magrans, s/n 08193 Bellaterra (Barcelona), Spain

2) Institut d'Estudis Espacials de Catalunya (IEEC)

Campus UAB, Carrer de Can Magrans, s/n 08193 Bellaterra (Barcelona), Spain

3) Laboratory for Theoretical Cosmology, Tomsk State University of Control Systems and Radioelectronics (TUSUR), 634050 Tomsk, Russia

4) Tomsk State Pedagogical University, 634061 Tomsk, Russia

elizalde@ieec.uab.es

\section{S.D. Odintsov}

1) Institució Catalana de Recerca i Estudis Avancats (ICREA), Barcelona, Spain

2) Instituto de Ciencias del Espacio (ICE/CSIC)

Campus UAB, Carrer de Can Magrans, s/n 08193 Bellaterra (Barcelona), Spain

3) Institut d'Estudis Espacials de Catalunya (IEEC)

Campus UAB, Carrer de Can Magrans, s/n 08193 Bellaterra (Barcelona), Spain

4) Laboratory for Theoretical Cosmology, Tomsk State University of Control Systems and Radioelectronics (TUSUR), 634050 Tomsk, Russia

5) Tomsk State Pedagogical University, 634061 Tomsk, Russia

odintsov@ieec.uab.es

\section{E.O. Pozdeeva}

Skobeltsyn Institute of Nuclear Physics, Lomonosov Moscow State University, Leninskiye Gory 1, 119991, Moscow, Russia

pozdeeva@www-hep.sinp.msu.ru

S.Yu. Vernov

Skobeltsyn Institute of Nuclear Physics, Lomonosov Moscow State University, Leninskiye Gory 1, 119991, Moscow, Russia

svernov@theory.sinp.msu.ru

The cosmological dynamics of a non-locally corrected gravity theory, involving a power of the inverse d'Alembertian, is investigated. Casting the dynamical equations into local form, the fixed points of the models are derived, as well as corresponding de Sitter and power-law solutions. Necessary and sufficient conditions on the model parameters for the existence of de Sitter solutions are obtained. The possible existence of power-law solutions is investigated, and it is proven that models with de Sitter solutions have no power-law solutions. A model is found, which allows to describe the matter-dominated phase of the Universe evolution.

Keywords: Non-local gravity, Gauss-Bonnet term, non-minimal coupling, exact solution 


\section{Introduction}

Reliable astronomical data support the existence of four distinct epochs of the Universe global evolution: inflation, a radiation dominated era, a matter dominated one and the present dark energy epoch. Initial inflation and dark energy domination are both characterized by an accelerated expansion of the Universe with almost constant Hubble parameter $H$. The other epochs of the Universe evolution are described by power-law solutions with $H=J / t$, where $J$ is a positive constant. In General Relativity, power-law solutions with $H=J / t$ correspond to models with a perfect fluid whose EoS parameter reads $w_{\mathrm{m}}=-1+2 /(3 J)$. As a consequence, the radiation dominated epoch corresponds to solutions with $J=1 / 2$, whereas the matter dominated one corresponds to solutions such that $J=2 / 3$. When one addresses the issue to consider new modified gravity models, it is therefore important to check for the existence of de Sitter and power-law solutions in the discussed models.

There are two basic motivations which lead cosmologists to modify gravity. The first one is an attempt to connect gravity with quantum physics, at least in a perturbative way, by including quantum correction terms to Einstein's equations. The second is an interest to describe the Universe evolution in a more natural way, without the dark energy and the dark matter components, which turn out to be avoidable in the modified models.

In string theory inspired models that include extra dimensions the Gauss-Bonnet term arises naturally [1/2]3. Such types of models allow to describe different epochs of the cosmological evolution. In particular, Gauss-Bonnet gravity models are actively used to investigate either the dark energy domination era 445|6/7/89, the inflation period [1011], or these two stages of the Universe evolution under the same scheme [1213. The scalar-Gauss-Bonnet gravity models allow to reconstruct the Universe expansion history, in which the matter dominated era makes a transition towards the cosmic acceleration epoch [6]. The $f(R)$-Gauss-Bonnet gravity, described by an analytic function of the Ricci scalar $R$ and the Gauss-Bonnet invariant, is being actively investigated as well. In particular, the Noether symmetry approach that allows to find out exact solutions has been developed in [14, the Newtonian and post Newtonian limits have been analyzed in [15. Note that both inflationary and dark energy evolutions can be described in the context of the $f(R)$-Gauss-Bonnet gravity [1617.

Another type of intensely investigated, modified gravity models is nonlocal gravity [18. Most of the non-local cosmological models are inspired by string theory [19] or by quantum field theory [20|21|22]. Usually, nonlocal models include an analytic function of either the d'Alembertian operator $\square$ or the inverse d'Alembertian operator $\square^{-1}$. Note that models including $F\left(\square R, \square^{2} R, \ldots, \square^{-1} R, \square^{-2} R, \ldots\right)$ have been investigated as well 2324 .

A model that includes a non-local term as a function of the $\square^{-1}$ operator: $R f\left(\square^{-1} R\right)$ has been proposed in $[20$, to explain current cosmic acceleration with- 
out dark energy (see also [25 26 27]). This class of non-local gravity models can be reformulated as a class of local model with additional scalar fields non-minimally coupled to gravity 28. It has been shown that the non-local and local formulations give the same solutions for the linear perturbations as long as the initial conditions are set the same [29. The relation between the original non-local equations and their biscalar-tensor representation in the context of an acausality problem has been discussed in 30. The difference between these formulations has been emphasized in [26]. The ensuing cosmology describing the four basic epochs and the perturbation analysis were studied in 31. Structure formation in this non-locally modified gravity model is being actively discussed 29132133 . The most studied model of this type is characterized by an exponential function $f\left(\square^{-1} R\right)=f_{0} e^{\alpha\left(\square^{-1} R\right)}$, where $f_{0}$ and $\alpha$ are real parameters $2328 / 3134351363738$. An explicit mechanism to screen the cosmological constant in these models has been investigated in 3435136. Its local formulation, including an additional Gauss-Bonnet term, has been explored in [39].

Another type of non-local infrared modifications of general relativity that includes the $\square^{-1}$ operator squared has been proposed in [40] to describe the dark energy dominated epoch. Cosmological perturbations and structure formation in these models have been studied in [41] (see also [42]). It has been shown that they allow to describe the $\mathrm{CMB}, \mathrm{BAO}$, and supernova data and fit these datasets at the same confidence level as $\Lambda \mathrm{CDM}$ [4344]. In the paper [45, non-local models with the Gauss-Bonnet term of a quite general form have been proposed, and accelerating cosmological solutions have been studieda. Also, a localization procedure that transforms a non-local model with the inverse d'Alambert operator acting on the Gauss-Bonnet term into a model of string-inspired scalar-Gauss-Bonnet gravity has been proposed in [45. In [12] this model has been modified by changing the $\square^{-1}$ operator for a $\left(\square-m^{2}\right)^{-1}$ operator, where $m$ is a new massive parameters. This modification allows to obtain de Sitter solutions with constant scalar fields that are absent in the original model [45].

In the present paper, we will continue to investigate the class of non-local models proposed in [45, and check for the existence of de Sitter and power-law solutions. We will find the conditions on the parameters of the model that are both necessary and sufficient for the existence of de Sitter solutions. We will also look for powerlaw solutions and prove that models with de Sitter solutions have no power-law solutions, whereas models without de Sitter solutions can have power-law ones. This result is important in relation with the characterization of the different epochs of the Universe evolution, as described above. Both de Sitter and power-law solutions are found in analytic form.

a Note that there is another class of non-local models with Gauss-Bonnet term, which includes analytic functions of the d'Alembertian operator [46]. 


\section{Non-local models with the Gauss-Bonnet term and their local formulation}

\subsection{Action}

Let us consider the following non-local model with Gauss-Bonnet term $\mathcal{G}$ :

$$
S_{N L}=\int d x^{4} \sqrt{-g}\left[\frac{M_{\mathrm{Pl}}^{2}}{16 \pi} R+C \mathcal{G}^{n_{1}} \square^{-n_{2}} \mathcal{G}^{n_{3}}-\Lambda\right],
$$

where $M_{\mathrm{Pl}}$ is the Planck mass, $C$ and $\Lambda$ are constants, $n_{k}$ natural numbers, the d'Alembertian operator in the metric $g_{\mu \nu}$, with determinant $g$, and the GaussBonnet term is given by

$$
\mathcal{G}=R^{2}-4 R_{\mu \nu} R^{\mu \nu}+R_{\mu \nu \alpha \beta} R^{\mu \nu \alpha \beta} .
$$

Using a localization procedure that is analogous to one proposed in 28 45], we introduce scalar fields and get the corresponding local model

$$
S_{L}=\int d x^{4} \sqrt{-g}\left[\frac{M_{\mathrm{Pl}}^{2}}{16 \pi} R+C \mathcal{G}^{n_{1}} \phi+\xi\left(\square^{n_{2}} \phi-\mathcal{G}^{n_{3}}\right)-\Lambda\right] .
$$

When $n_{2}>1$, the scalars fields $\xi$ and $\phi$ can be expressed in terms of $2 n_{2}$ scalar fields $\xi_{j}$ and $\phi_{j}$ that satisfy second order equations. Indeed,

$S_{L}=\int d x^{4} \sqrt{-g}\left[\frac{M_{P l}^{2}}{16 \pi} R+C \mathcal{G}^{n_{1}} \phi_{n_{2}}+\xi_{1}\left(\square \phi_{1}-\mathcal{G}^{n_{3}}\right)+\sum_{j=2}^{n_{2}} \xi_{j}\left(\square \phi_{j}-\phi_{j-1}\right)-\Lambda\right]$.

Varying this action under $\xi_{j}$, we get

$$
\begin{aligned}
\square \phi_{1}=\mathcal{G}^{n_{3}}, & j=1, \\
\square \phi_{j}=\phi_{j-1}, & j=2, \ldots, n_{2} .
\end{aligned}
$$

Also, this action can be presented in the form

$$
S_{L 1}=\int d x^{4} \sqrt{-g}\left[\frac{M_{P l}^{2}}{16 \pi} R+C \mathcal{G}^{n_{1}} \phi_{n_{2}}-\xi_{1} \mathcal{G}^{n_{3}}+\sum_{j=1}^{n_{2}} \phi_{j} \square \xi_{j}-\sum_{j=1}^{n_{2}-1} \xi_{j+1} \phi_{j}-\Lambda\right],
$$

and variation under $\phi_{J}$ leads to

$$
\begin{aligned}
\square \xi_{n_{2}}=-C \mathcal{G}^{n_{1}}, & j=n_{2}, \\
\square \xi_{j}=\xi_{j+1}, & j=1, \ldots, n_{2}-1 .
\end{aligned}
$$

The action $S_{L}$ can be linearized with respect to the Gauss-Bonnet term, by adding one more scalar field to it (see [5]). Let us consider the part of action $S_{L}$ that includes the Gauss-Bonnet term, namely

$$
S_{f G B}=\int d x^{4} \sqrt{-g}\left[C \mathcal{G}^{n_{1}} \phi_{n_{2}}-\xi_{1} \mathcal{G}^{n_{3}}\right]
$$

To linearize this action with respect to $\mathcal{G}$ we introduce a scalar field $\sigma$ and

$$
f(\sigma)=C \sigma^{n_{1}} \phi_{n_{2}}-\xi_{1} \sigma^{n_{3}}
$$


and thus get the following equivalent action:

$$
\begin{aligned}
& S_{G B \sigma}=\int d x^{4} \sqrt{-g}\left[\frac{d f}{d \sigma}(\mathcal{G}-\sigma)+f\right]= \\
& \quad=\int d x^{4} \sqrt{-g}\left[\left(n_{1} C \sigma^{n_{1}-1} \phi_{n_{2}}-n_{3} \xi_{1} \sigma^{n_{3}-1}\right)(\mathcal{G}-\sigma)+C \sigma^{n_{1}} \phi_{n_{2}}-\xi_{1} \sigma^{n_{3}}\right] .
\end{aligned}
$$

Varying over $\sigma$, one obtains that $\sigma=\mathcal{G}$ and the action $S_{f G B}$. Note that the scalar field $\sigma$ is not dynamical, because it has no kinetic energy term.

As a consequence, the initial action $S_{N L}$ can be written in the following scalartensor form:

$$
S=\int d x^{4} \sqrt{-g}\left[\frac{M_{P l}^{2}}{16 \pi} R+F \mathcal{G}-V-\sum_{k=1}^{n_{2}} g^{\mu \nu} \partial_{\mu} \xi_{k} \partial_{\nu} \phi_{k}\right],
$$

where we use the following re-designations

$$
\begin{aligned}
& F=n_{1} C \sigma^{n_{1}-1} \phi_{n_{2}}-n_{3} \xi_{1} \sigma^{n_{3}-1}, \\
& V=-C \sigma^{n_{1}} \phi_{n_{2}}\left(1-n_{1}\right)-\xi_{1} \sigma^{n_{3}}\left(n_{3}-1\right)+\sum_{k=1}^{n_{2}-1} \xi_{k+1} \phi_{k}+\Lambda .
\end{aligned}
$$

A similar action with one scalar field non-minimally coupled with the Gauss-Bonnet term has been studied in [5]6].

Varying the local action $S$ thus obtained, we get the following equations

$$
\begin{aligned}
& {\left[\frac{M_{P l}^{2}}{16 \pi}-4 \square F\right]\left[R^{\mu \nu}-\frac{1}{2} g^{\mu \nu} R\right]+\frac{g^{\mu \nu}}{2}\left(\sum_{k=1}^{n_{2}} \partial_{\sigma} \phi_{k} \partial^{\sigma} \xi_{k}\right)-\sum_{k=1}^{n_{2}} \partial^{\mu} \phi_{k} \partial^{\nu} \xi_{k}=} \\
& =F\left[\frac{1}{2} g^{\mu \nu} \mathcal{G}-2 R R^{\mu \nu}+4 R_{\rho}^{\mu} R^{\nu \rho}-2 R^{\mu \rho \sigma \tau} R_{\rho \sigma \tau}^{\nu}+4 R^{\mu \rho \sigma \nu} R_{\rho \sigma}\right]-\frac{g^{\mu \nu}}{2} V+ \\
& 2 R\left(D^{\mu} D^{\nu} F\right)-4\left(D_{\rho} D^{\mu} F\right) R^{\nu \rho}-4\left(D_{\rho} D^{\nu} F\right) R^{\mu \rho}+ \\
& 4 g^{\mu \nu}\left(D_{\rho} D_{\sigma} F\right) R^{\rho \sigma}-4\left(D_{\rho} D_{\sigma} F\right) R^{\mu \rho \nu \sigma} .
\end{aligned}
$$

Variation under $\sigma$ leads to

$$
\left[n_{1}\left(n_{1}-1\right) C \sigma^{n_{1}-2} \phi_{n_{2}}+n_{3}\left(n_{3}-1\right) \xi_{1} \sigma^{n_{3}-2}\right](\mathcal{G}-\sigma)=0, \quad \Rightarrow \quad \mathcal{G}=\sigma .
$$

Let us consider the trace of Eq. (11). After setting $R^{\mu \rho \sigma \nu}=R^{\nu \rho \mu \sigma}$ and $g_{\mu \nu} R^{\nu \rho \mu \sigma} R_{\rho \sigma}=R_{\mu}^{\rho \mu \sigma} R_{\rho \sigma}=R^{\rho \sigma} R_{\rho \sigma}$, we get

$$
g_{\mu \nu} F\left[\frac{1}{2} g^{\mu \nu} \mathcal{G}-2 R R^{\mu \nu}+4 R_{\rho}^{\mu} R^{\nu \rho}-2 R^{\mu \rho \sigma \tau} R_{\rho \sigma \tau}^{\nu}+4 R^{\mu \rho \sigma \nu} R_{\rho \sigma}\right]=0
$$

Using $R^{\mu \nu} D_{\mu} D_{\nu} F=R \square F$ and

$$
-8\left(D_{\rho} D^{\nu} F\right) R_{\nu}^{\rho}+16\left(D_{\rho} D_{\sigma} F\right) R^{\rho \sigma}-4\left(D_{\rho} D_{\sigma} F\right) R_{\nu}^{\rho \nu \sigma}=4\left(D_{\rho} D_{\sigma} F\right) R^{\rho \sigma},
$$

we obtain the trace equation:

$$
\frac{M_{P l}^{2}}{16 \pi} R-\left(\sum_{k=1}^{n_{2}} \partial_{\sigma} \phi_{k} \partial^{\sigma} \xi_{k}\right)-2 V-R(\square F)=0 .
$$




\section{Cosmological equations}

\subsection{The FLRW metric}

Let us consider the spatially flat FLRW universe with metric

$$
d s^{2}=-d t^{2}+a^{2}(t)\left(d x_{1}^{2}+d x_{2}^{2}+d x_{3}^{2}\right)
$$

From this metric, one gets $(i, j, m, l=1,2,3)$ :

$$
\begin{gathered}
R^{i 0 j 0}=R^{0 i 0 j}=-R^{0 i j 0}=-R^{i 00 j}=-\frac{\left[\dot{H}+H^{2}\right]}{a^{2}} \delta_{i j}, R^{i j m l}=\frac{H^{2}}{a^{4}}\left(\delta_{i m} \delta_{l j}-\delta_{i l} \delta_{m j}\right), \\
\Gamma_{i j}^{0}=a^{2} H \delta_{i j}, \quad \Gamma_{0 j}^{i}=\Gamma_{j 0}^{i}=H \delta_{j}^{i}, \\
R^{00}=-3\left(\dot{H}+H^{2}\right), \quad R^{i j}=\frac{\left[\dot{H}+3 H^{2}\right]}{a^{2}} \delta_{i j}, \\
R=6\left(\dot{H}+2 H^{2}\right), \quad \mathcal{G}=24 H^{2}\left(\dot{H}+H^{2}\right),
\end{gathered}
$$

where the Hubble parameter is $H=\dot{a} / a$, and the dots mean time derivatives.

We will assume that all scalar fields depend on time only, and thus get the following expressions, including components equations

$$
\begin{gathered}
\square F=-3 H \dot{F}-\ddot{F}, \\
D_{\rho} D_{\sigma} F=\partial_{\rho \sigma}^{2} F-\Gamma_{\rho \sigma}^{j} \partial_{j} F,
\end{gathered}
$$

from where

$$
\begin{gathered}
D^{i} D^{i} F=-\frac{H}{a^{2}} \dot{F}, \quad D_{i} D_{i} F=-a^{2} H \dot{F}, \\
D^{0} D^{0} F=D_{0} D_{0} F=\ddot{F}, \quad D^{i} D^{0} F=D^{0} D^{i} F=0 .
\end{gathered}
$$

\subsection{Field and Friedmann equations}

The field equations (3) and (4) in the FLRW metric (15) have the following form

$$
\ddot{\phi}_{k}=-3 H \dot{\phi}_{k}+\mathcal{G} \frac{\partial F}{\partial \xi_{k}}-\frac{\partial V}{\partial \xi_{k}}, \quad \ddot{\xi}_{k}=-3 H \dot{\xi}_{k}+\mathcal{G} \frac{\partial F}{\partial \phi_{k}}-\frac{\partial V}{\partial \phi_{k}} .
$$

Eqs. (11) in the FLRW metric read as follows

$$
\begin{gathered}
3 H^{2} \frac{M_{P l}^{2}}{16 \pi}-\frac{1}{2} \sum_{k=1}^{n_{2}}\left(\dot{\phi}_{k} \dot{\xi}_{k}\right)-\frac{1}{2} V=-12 H^{3} \dot{F} \\
-\left(3 H^{2}+2 \dot{H}\right) \frac{M_{P l}^{2}}{16 \pi}-8 H\left(H^{2}+\dot{H}\right) \dot{F}-4 H^{2} \ddot{F}-\frac{1}{2} \sum_{k=1}^{n_{2}} \dot{\phi}_{k} \dot{\xi}_{k}+\frac{V}{2}=0 .
\end{gathered}
$$


Subtracting (22) from (21), we get

$$
8 H^{3} \dot{F}-4 \square F H^{2}+\frac{3 M_{P l}^{2}}{8 \pi} H^{2}+8 H \dot{F} \dot{H}+\frac{M_{P l}^{2}}{8 \pi} \dot{H}-V=0 .
$$

Note that Eqs. (22) and (23) are third order differential equations with respect to the Hubble parameter.

Let us show that Eq. (22) is a consequence of Eqs. (20) and (21). Differentiating Eq. (21) with respect to $t$, we obtain

$$
6 H \dot{H} \frac{M_{P l}^{2}}{16 \pi}-\frac{1}{2}\left[\sum_{k=1}^{n_{2}}\left(\ddot{\phi}_{k} \dot{\xi}_{k}+\dot{\phi}_{k} \ddot{\xi}_{k}\right)+\dot{V}\right]=-36 H^{2} \dot{H} \dot{F}-12 H^{3} \ddot{F} .
$$

Taking into account Eqs. (20) we modify Eq. (24), using

$$
\sum_{k=1}^{n_{2}}\left(\ddot{\phi}_{k} \dot{\xi}_{k}+\dot{\phi}_{k} \ddot{\xi}_{k}\right)+\dot{V}=-6 H \sum_{k=1}^{n_{2}}\left(\dot{\phi}_{k} \dot{\xi}_{k}\right)+\mathcal{G} \dot{F} .
$$

Dividing Eq. (24) by $3 H$ and adding it up with Eq. (21), we get Eq. (22). So, to obtain solutions of the models we are here considering, it is enough to solve the corresponding field equations together with Eq. (21).

\section{Search for de Sitter solutions}

If the Hubble parameter is a constant: $H=H_{0}$, then the Gauss-Bonnet term reads $\mathcal{G}=24 H_{0}^{4} \equiv \mathcal{G}_{0}$ and $\sigma=\mathcal{G}_{0}$. As a consequence, the corresponding field equations (3) get transformed into the following system of linear first order differential equations, with constant coefficients,

$$
\begin{aligned}
& \dot{\phi}_{1}=\psi_{1}, \\
& \dot{\psi}_{1}=-3 H_{0} \psi_{1}-\mathcal{G}_{0}^{n_{3}}, \\
& \dot{\phi}_{j}=\psi_{j}, \quad j=2, \ldots, n_{2}, \\
& \dot{\psi}_{j}=-3 H_{0} \psi_{j}-\phi_{j-1}, \quad j=2, \ldots, n_{2} .
\end{aligned}
$$

The system (26) has the following solution

$$
\phi_{j}=P_{j}(t) e^{-3 H_{0} t}-\frac{\mathcal{G}_{0}^{n_{3}}}{j !\left(3 H_{0}\right)^{j}} t^{j}+\tilde{P}_{j}(t),
$$

where $P_{j}(t)$ and $\tilde{P}_{j}(t)$ are $(j-1)$-degree polynomials of $t$ with coefficients that include $2 j$ arbitrary parameters.

Analogously, the system (4) acquires the following form

$$
\begin{aligned}
\ddot{\xi}_{j}+3 H_{0} \dot{\xi}_{j}+\xi_{j+1} & =0, \quad j=1, \ldots, n_{2}-1, \\
\ddot{\xi}_{n_{2}}+3 H_{0} \dot{\xi}_{n_{2}}-C \mathcal{G}_{0}^{n_{1}} & =0,
\end{aligned}
$$

and the solution reads

$$
\xi_{j}=Q_{j}(t) e^{-3 H_{0} t}+\frac{C \mathcal{G}_{0}^{n_{1}}}{\left(n_{2}-j+1\right) !\left(3 H_{0}\right)^{n_{2}-j+1}} t^{\left(n_{2}-j+1\right)}+\tilde{Q}_{j}(t),
$$


E. Elizalde, S.D. Odintsov, E.O. Pozdeeva, S.Yu. Vernov

where $Q_{j}(t)$ and $\tilde{Q}_{j}(t)$ are polynomials in $t$ of degree $\left(n_{2}-j\right)+1$.

To check for the existence of de Sitter solutions, one must substitute the solutions of the field equations thus obtained into Eqs. (21) and (23). In the case when $n_{1}=n_{2}=n_{3}=1$ the Sitter solutions have been found in [45]. In this paper, we consider the case $n_{2}=2$.

The field equations

$$
-\ddot{\phi}_{1}-3 H_{0} \dot{\phi}_{1}=\mathcal{G}_{0}^{n_{3}}, \quad-\ddot{\phi}_{2}-3 H_{0} \dot{\phi}_{2}=\phi_{1}
$$

and

$$
-\ddot{\xi}_{2}-3 H_{0} \dot{\xi}_{2}=-C \mathcal{G}_{0}^{n_{1}}, \quad-\ddot{\xi}_{1}-3 H_{0} \dot{\xi}_{1}=\xi_{2}
$$

have the following solutions:

$$
\begin{gathered}
\phi_{1}=A_{1} e^{-3 H_{0} t}-\frac{\mathcal{G}_{0}^{n_{3}}}{3 H_{0}} t+B_{1} \\
\phi_{2}=\left(\frac{A_{1}}{3 H_{0}} t+A_{2}\right) e^{-3 H_{0} t}+\frac{\mathcal{G}_{0}^{n_{3}}}{18 H_{0}^{2}} t^{2}-\left(\frac{\mathcal{G}_{0}^{n_{3}}}{27 H_{0}^{3}}+\frac{B_{1}}{3 H_{0}}\right) t+B_{2}, \\
\xi_{1}=\left(\frac{C_{1}}{3 H_{0}} t+C_{2}\right) e^{-3 H_{0} t}-\frac{C \mathcal{G}_{0}^{n_{1}}}{18 H_{0}^{2}} t^{2}+C\left(\frac{\mathcal{G}_{0}^{n_{1}}}{27 H_{0}^{3}}-\frac{D_{1}}{3 H_{0}}\right) t+D_{2} \\
\xi_{2}=C_{1} e^{-3 H_{0} t}+C \frac{\mathcal{G}_{0}^{n_{1}}}{3 H_{0}} t+C D_{1}
\end{gathered}
$$

where $A_{i}, B_{i}, C_{i}$, and $D_{i}$ are integration constants.

Substituting these expressions into Eq. (21),

$$
3 H_{0}^{2} \frac{M_{P l}^{2}}{16 \pi}-\frac{1}{2}\left(\sum_{k=1}^{n_{2}} \dot{\phi}_{k} \dot{\xi}_{k}\right)-\frac{1}{2} V=-12 H_{0}^{3} \dot{F}
$$

we see that this equation can be satisfied only if $n_{1}+n_{3}=4$. Also, we get the following restriction to the integration constants

$$
\begin{gathered}
A_{1}=0, \quad B_{1}=-\frac{331776\left(n_{1}-2\right) H_{0}^{16-8 n_{1}} D_{1}+24^{n_{1}} 442368 H_{0}^{14-4 n_{1}}}{\left(n_{1}-2\right)}, \\
C_{1}=0, \quad C_{2}=-\frac{24^{2 n_{1}}\left(2 n_{1}-1\right) A_{2} C H_{0}^{8\left(n_{1}-2\right)}}{331776\left(2 n_{1}-7\right)} .
\end{gathered}
$$

These restrictions are not valid for $n_{1}=2$.

Also, we have the additional to connect the values of the parameters of the solutions, with $\Lambda$

$$
\begin{aligned}
\Lambda & =-\frac{3 H_{0}^{2} M_{P l}^{2}}{8 \pi}-\frac{8192 C\left(13 n_{1}+4\right) H_{0}^{12}}{\left(n_{1}-2\right)} \\
& +24^{-n_{1}} 331776 D_{2}\left(n_{1}-3\right) H_{0}^{16-4 n_{1}}+24^{n_{1}} C B_{2}\left(n_{1}-1\right) H_{0}^{4 n_{1}} \\
& -\frac{24^{-n_{1}} 73728\left(5 n_{1}-4\right) C D_{1} H_{0}^{14-4 n_{1}}}{n_{1}-2}-24^{-2 n_{1}} 331776 C D_{1}^{2} H_{0}^{16-8 n_{1}} .
\end{aligned}
$$


Consequently, the value of $\Lambda$ fixes the value of one of the integration constants: $B_{2}$ for $n_{1}=3$ or $D_{2}$ for $n_{1}=1$.

Summing up, we do get explicitly de Sitter solutions for models with $n_{1}=1$, $n_{2}=2, n_{3}=3$ and $n_{1}=3, n_{2}=2, n_{3}=1$. And we have also discovered that models with $n_{2}=2$ and other values of $n_{1}$ and $n_{3}$ do not have de Sitter solutions $\mathrm{b}$. In the next section we will check the existence of power-low solutions for those models.

\section{Power Law solutions}

The search of power-law solutions with $H=J / t$ is more complicated. We consider the case when $n_{1}$ or $n_{3}$ is equal to 1 . If $n_{1}=1$ and $n_{3}=1$, then

$$
V=\xi_{2} \phi_{1}+\Lambda, \quad F=C \phi_{2}-\xi_{1},
$$

with the following form for the field equations

$$
\begin{gathered}
\square \phi_{1}=\mathcal{G}, \quad \square \phi_{2}=\phi_{1}, \\
\square \xi_{2}=-C \mathcal{G}, \quad \square \xi_{1}=\xi_{2},
\end{gathered}
$$

where $\mathcal{G}=24(J-1) J^{3} / t^{4}$.

Using these formulas, we immediately obtain the form of Eq. (23)

$$
-2\left(3 H^{2}+\dot{H}\right) \frac{M_{P l}^{2}}{16 \pi}-8 H\left(H^{2}+\dot{H}\right)\left(C \dot{\phi}_{2}-\dot{\xi}_{1}\right)+4\left(C \phi_{1}-\xi_{2}\right) H^{2}+\xi_{2} \phi_{1}=0 .
$$

The model with $n_{1}=1$ and $n_{3}=1$ yields power law solutions with $H=J / t$ at $J=2 / 3$ and $J=3$. These solutions exist at $\Lambda=0$ only. The corresponding scalar fields admit two types of expressions. The first type of solutions corresponds to

$$
\begin{aligned}
\phi_{1} & =-\frac{C_{1} t^{-3 J+1}}{3 J-1}+4 \frac{J^{3}}{t^{2}}-\frac{M_{P l}^{2}}{32 \pi} \frac{(3 J+1)}{J C(J-1)}, \\
\phi_{2} & =\frac{M_{P l}^{2}}{64 \pi} \frac{t^{2}}{J C(J-1)}-\frac{C_{3} t^{-3 J+1}}{C(3 J-1)}-\frac{C_{1} t^{3-3 J}}{6\left(3 J^{2}-4 J+1\right)}-4 \frac{J^{3} \ln (t)}{3 J-1}+C_{4}, \\
\xi_{1} & =4 \frac{C J^{3}}{3 J-1} \ln (t)-\frac{C_{3} t^{-3 J+1}}{3 J-1}+C_{2}, \\
\xi_{2} & =-4 \frac{C J^{3}}{t^{2}}
\end{aligned}
$$

where in the case $J=2 / 3, C_{1}=\frac{7168}{729 C_{3}}$, while in the case $J=3$, either $C_{1}=0$ or $C_{3}=0$.

${ }^{\mathrm{b}}$ Straightforward substitution of the field expressions when $n_{1}=n_{3}=2$ already proves the absence of the de Sitter solutions in this case. 
Another type of solutions, with the same Hubble parameter, is given by

$$
\begin{aligned}
& \phi_{1}=4 \frac{J^{3}}{t^{2}}, \\
& \phi_{2}=-\frac{C_{3} t^{-3 J+1}}{3 J-1}-\frac{4 J^{3}}{3 J-1} \ln (t)+C_{4}, \\
& \xi_{1}=4 \frac{C J^{3} \ln (t)}{3 J-1}-\frac{C C_{3} t^{-3 J+1}}{3 J-1}-\frac{M_{P l}^{2}}{64 \pi} \frac{t^{2}}{J(J-1)}-\frac{t^{3-3 J} C_{1}}{6\left(3 J^{2}-4 J+1\right)}+C_{2}, \\
& \xi_{2}=-\frac{C_{1} t^{-3 J+1}}{3 J-1}-4 \frac{C J^{3}}{t^{2}}+\frac{M_{P l}^{2}}{32 \pi} \frac{(3 J+1)}{J(J-1)},
\end{aligned}
$$

where in the case $J=2 / 3$ we have the additional condition $C_{1}=-\frac{7168 C}{729 C_{3}}$, while in the case $J=3$, either $C_{3}=0$ or $C_{1}=0$. Other $C_{i}$ are arbitrary constants. Note that the form of the solutions obtained excludes a few values of $J$, which must be checked separately. There is no solution with $J=1 / 3$. In the case $J=1$ that corresponds to $\mathcal{G}=0$ we do not seek solutions.

Let us consider the case $n_{1}=1$ and $n_{3}>1$. The expressions for $\phi_{2}(t)$ do not include a logarithmic term, whereas $\xi_{1}(t)$ has a logarithmic term, which cannot be remove by the choice of integration constants. Substituting the field expressions thus obtained into Eq. (21), we get that the logarithmic term of the equation has the following coefficient

$$
\frac{24^{n_{3}}\left(2\left(n_{3}-1\right)\right) C J^{3}(J-1)^{n_{3}} J^{3 n_{3}}\left(4 n_{3}+J-1\right)}{(J-1)(3 J-1)} t^{-4 n_{3}} .
$$

This coefficient is equal to zero only for $J=-4 n_{3}+1<0$. Such case is not interesting because the corresponding Hubble parameter is strictly negative. As a consequence, the model with $n_{1}=1$ and $n_{3}>1$ admits power-law solutions only for values of $J$ such that a denominator of some field expression is zero. At the same time, there is no solution with $J=1 / 3$ for models with $n_{1}=1$ and $n_{3}>1$.

\section{Conclusion}

In this paper we have analyzed a set of non-local cosmological models with the Gauss-Bonnet term given by the action (1). We have shown how this action can be transformed into a local one and, after that, we have looked for de Sitter and power-law solutions for the corresponding models in the specific case $n_{2}=2$.

The result we have obtained is that de Sitter solutions exist only in these two cases: for $n_{1}=1$ and $n_{3}=3$, or for $n_{1}=3$ and $n_{3}=1$. We have seen that these models yield no power-law solutions. Moreover, we have shown that, if $n_{1}=1$ and $n_{3}>1$ (or $n_{1}>1$ and $n_{3}=1$, respectively), then power-law solutions do not exist. In the case $n_{1}=n_{3}=1$, power-law solutions with $H=J / t$ exist only for $J=2 / 3$ and $J=3$. Therefore, the model with $n_{1}=1, n_{2}=2$, and $n_{3}=1$, without additional matter, is suitable in order to describe the matter-dominated phase of the Universe evolution that corresponds to $J=2 / 3$. 
As an extension of this work, we plan to consider the stability of the de Sitter solutions obtained and the possibility to describe dark energy in models of this type. Also, it is naturally assumed that non-local modifications of gravity can be important at the very high energy scales that correspond to inflation. In this respect, note that the models here with de Sitter solutions may serve for the construction of eternal inflation. It is thus an interesting and non-trivial problem to try to generalize the known inflationary scenarios by adding a non-local gravity term 28147. We plan to consider the possibility to use the here considered models of non-local GaussBonnet gravity for the generalization of local inflationary scenarios.

\section{Acknowledgements}

This work has been partially supported by MINECO (Spain), Project FIS201676363-P, and by the CPAN Consolider Ingenio 2010 Project. Research of E.P. and S.V. is supported in part by the RFBR grant 18-52-45016.

\section{References}

[1] I. Antoniadis, J. Rizos and K. Tamvakis, Singularity-free cosmological solutions of the superstring effective action, Nucl. Phys. B 415 (1994) 497 hep-th/9305025.

[2] T. Torii, H. Yajima and K. i. Maeda, Dilatonic black holes with Gauss-Bonnet term, Phys. Rev. D 55 (1997) 739 gr-qc/9606034.

[3] S. Kawai, M. a. Sakagami and J. Soda, Instability of one loop superstring cosmology, Phys. Lett. B 437 (1998) 284 gr-qc/9802033;

S. Kawai and J. Soda, Evolution of fluctuations during graceful exit in string cosmology, Phys. Lett. B 460 (1999) 41 gr-qc/9903017.

[4] S. Nojiri and S. D. Odintsov, Modified Gauss-Bonnet theory as gravitational alternative for dark energy, Phys. Lett. B 631, 1 (2005) hep-th/0508049;

S. Nojiri, S. D. Odintsov and M. Sasaki, Gauss-Bonnet dark energy, Phys. Rev. D 71 (2005) 123509 hep-th/0504052.

[5] G. Cognola, E. Elizalde, S. Nojiri, S. D. Odintsov and S. Zerbini, Dark energy in modified Gauss-Bonnet gravity: Late-time acceleration and the hierarchy problem, Phys. Rev. D 73 (2006) 084007 hep-th/0601008.

[6] G. Cognola, E. Elizalde, S. Nojiri, S. Odintsov and S. Zerbini, String-inspired GaussBonnet gravity reconstructed from the universe expansion history and yielding the transition from matter dominance to dark energy, Phys. Rev. D 75 (2007) 086002 hep-th/0611198.

[7] M. Sami, A. Toporensky, P. V. Tretjakov and S. Tsujikawa, The Fate of (phantom) dark energy universe with string curvature corrections, Phys. Lett. B 619 (2005) 193 hep-th/0504154.

[8] E. Elizalde, R. Myrzakulov, V. V. Obukhov and D. Saez-Gomez, LambdaCDM epoch reconstruction from $\mathrm{F}(\mathrm{R}, \mathrm{G})$ and modified Gauss-Bonnet gravities, Class. Quant. Grav. 27 (2010) 095007 arXiv:1001.3636 [gr-qc]].

[9] M. Benetti, S. Santos da Costa, S. Capozziello, J. S. Alcaniz and M. De Laurentis, Observational constraints on Gauss-Bonnet cosmology, arXiv:1803.00895 [gr-qc].

[10] Z. K. Guo and D. J. Schwarz, Slow-roll inflation with a Gauss-Bonnet correction, Phys. Rev. D 81 (2010) 123520 arXiv:1001.1897 [hep-th]]. 
[11] V. K. Oikonomou, Autonomous dynamical system approach for inflationary GaussBonnet modified gravity, Int. J. Mod. Phys. D 27 (2018) 1850059 arXiv:1711.03389 [gr-qc]].

[12] G. Cognola, E. Elizalde, S. Nojiri, S.D. Odintsov, and S. Zerbini, One-loop effective action for non-local modified Gauss-Bonnet gravity in deSitter space, Eur. Phys. J. C 64 (2009) 483, arXiv:0905.0543

[13] C. van de Bruck, K. Dimopoulos, C. Longden and C. Owen, Gauss-Bonnet-coupled Quintessential Inflation, arXiv:1707.06839 [astro-ph.CO].

[14] S. Capozziello, M. De Laurentis and S.D. Odintsov, Noether Symmetry Approach in Gauss-Bonnet Cosmology, Mod. Phys. Lett. A 29 (2014) no.30, 1450164 arXiv:1406.5652 [gr-qc]].

[15] M. De Laurentis and A. J. Lopez-Revelles, Newtonian, Post Newtonian and Parameterized Post Newtonian limits of $f(R, G)$ gravity, Int. J. Geom. Meth. Mod. Phys. 11 (2014) 1450082 arXiv:1311.0206 [gr-qc]].

[16] M. De Laurentis, M. Paolella and S. Capozziello, Cosmological inflation in $F(R, \mathcal{G})$ gravity, Phys. Rev. D 91 (2015) 083531 arXiv:1503.04659 [gr-qc]].

[17] S. D. Odintsov, V. K. Oikonomou and S. Banerjee, Dynamics of Inflation and Dark Energy from $F(R, \mathcal{G})$ Gravity, arXiv:1807.00335 [gr-qc].

[18] N. Arkani-Hamed, S. Dimopoulos, G. Dvali and G. Gabadadze, Non-local modification of gravity and the cosmological constant problem, arXiv:hep-th/0209227;

D. Espriu, T. Multamaki, and E.C. Vagenas, Cosmological significance of one-loop effective gravity, Phys. Lett. B 628 (2005) 197, arXiv:gr-qc/0503033;

G. Calcagni, M. Montobbio, and G. Nardelli, Localization of nonlocal theories, Phys. Lett. B 662 (2008) 285, arXiv:0712.2237;

F.W. Hehl and B. Mashhoon, A formal framework for a nonlocal generalization of Einstein's theory of gravitation, Phys. Rev. D 79 (2009) 064028, arXiv:0902.0560;

G. Calcagni and G. Nardelli, Cosmological rolling solutions of nonlocal theories, Int. J. Mod. Phys. D 19 (2010) 329, arXiv:0904.4245;

G. Calcagni and G. Nardelli, Nonlocal gravity and the diffusion equation, Phys. Rev. D 82 (2010) 123518, arXiv:1004.5144;

L. Modesto, Super-renormalizable Quantum Gravity, Phys. Rev. D 86 (2012) 044005, arXiv:1107.2403;

A.O. Barvinsky, Serendipitous discoveries in nonlocal gravity theory, Phys. Rev. D 85 (2012) 104018, arXiv:1112.4340;

A.S. Koshelev and S.Yu. Vernov, On bouncing solutions in non-local gravity, Phys. Part. Nucl. 43 (2012) 666, arXiv:1202.1289;

T. Biswas, A.S. Koshelev, A. Mazumdar, and S.Yu. Vernov, Stable bounce and inflation in non-local higher derivative cosmology, J. Cosmol. Astropart. Phys. 1208 (2012) 024, arXiv:1206.6374;

L. Modesto and S. Tsujikawa, Non-local massive gravity, Phys. Lett. B 727 (2013) 48 [arXiv:1307.6968 [hep-th]];

B. Craps, T. De Jonckheere and A. S. Koshelev, Cosmological perturbations in non-local higher-derivative gravity, J. Cosmol. Astropart. Phys. 1411 (2014) 022 arXiv:1407.4982 [hep-th]];

L. Modesto and L. Rachwal, Super-renormalizable and finite gravitational theories, Nucl. Phys. B 889 (2014) 228 arXiv:1407.8036 [hep-th]];

A. S. Koshelev and S. Yu. Vernov, Cosmological Solutions in Nonlocal Models, Phys. Part. Nucl. Lett. 11 (2014) no.7, 960 arXiv:1406.5887] [gr-qc]];

V. Vardanyan, Y. Akrami, L. Amendola and A. Silvestri, On nonlocally interacting metrics, and a simple proposal for cosmic acceleration, J. Cosmol. Astropart. Phys. 
1803 (2018) 048 arXiv:1702.08908 [gr-qc]];

G. Narain and T. Li, Ultraviolet complete dark energy model, Phys. Rev. D 97 (2018) 083523 arXiv:1712.09054 [hep-th]];

G. Calcagni, L. Modesto and G. Nardelli, Initial conditions and degrees of freedom of non-local gravity, J. High Energy Phys. 1805 (2018) 087 arXiv:1803.00561 [hep-th]]; G. Calcagni, L. Modesto and G. Nardelli, Nonperturbative spectrum of nonlocal gravity, arXiv:1803.07848 [hep-th].

[19] I.Ya. Aref'eva, Nonlocal String Tachyon as a Model for Cosmological Dark Energy, AIP Conf. Proc. 826, p-Adic Mathematical Physics, eds. A.Yu. Khrennikov, Z. Rakich, I.V. Volovich, AIP, Melville, NY, 2006, pp. 301-311, arXiv:astro-ph/0410443; A.S. Koshelev, Non-local SFT Tachyon and Cosmology, J. High Energy Phys. 0704 (2007) 029, arXiv:hep-th/0701103;

I.Ya. Aref'eva, L.V. Joukovskaya, and S.Yu. Vernov, Bouncing and accelerating solutions in nonlocal stringy models J. High Energy Phys. 0707 (2007) 087, arXiv:hep-th/0701184;

I.Ya. Aref'eva and I.V. Volovich, Quantization of the Riemann Zeta-Function and Cosmology, Int. J. of Geom. Meth. Mod. Phys. 4 (2007) 881, arXiv:hep-th/0701284; J.E. Lidsey, Stretching the Inflaton Potential with Kinetic Energy, Phys. Rev. D 76 (2007) 043511, arXiv:hep-th/0703007;

G. Calcagni, M. Montobbio, and G. Nardelli, A route to nonlocal cosmology, Phys. Rev. D 76 (2007) 126001, arXiv:0705.3043;

N. Barnaby, T. Biswas, and J.M. Cline, p-adic Inflation, J. High Energy Phys. 0704 (2007) 056, arXiv:hep-th/0612230;

N. Barnaby and J.M. Cline, Large Nongaussianity from Nonlocal Inflation, J. Cosmol. Astropart. Phys. 0707 (2007) 017, arXiv:0704.3426;

I.Ya. Aref'eva, L.V. Joukovskaya, and S.Yu. Vernov, Dynamics in nonlocal linear models in the Friedmann-Robertson-Walker metric, J. Phys. A: Math. Theor. 41 (2008) 304003, arXiv:0711.1364;

D.J. Mulryne and N.J. Nunes, Diffusing non-local inflation: Solving the field equations as an initial value problem, Phys. Rev. D 78 (2008) 063519, arXiv:0805.0449; G. Calcagni, M. Montobbio, and G. Nardelli, Localization of nonlocal theories, Phys. Lett. B 662 (2008) 285, arXiv:0712.2237;

G. Calcagni and G. Nardelli, Nonlocal instantons and solitons in string models, Phys. Lett. B 669 (2008) 102 arXiv:0802.4395 [hep-th]];

L.V. Joukovskaya, Dynamics in nonlocal cosmological models derived from string field theory Phys. Rev. D 76 (2007) 105007, arXiv:0707.1545;

N. Barnaby and N. Kamran, Dynamics with Infinitely Many Derivatives: The Initial Value Problem, J. High Energy Phys. 0802 (2008) 008, arXiv:0709.3968;

G. Calcagni and G. Nardelli, Cosmological rolling solutions of nonlocal theories, Int. J. Mod. Phys. D 19 (2010) 329, arXiv:0904.4245;

$\mathrm{S} . Y u$. Vernov, Localization of nonlocal cosmological models with quadratic potentials in the case of double roots, Class. Quant. Grav. 27 (2010) 035006, arXiv:0907.0468; A.S. Koshelev and S.Yu. Vernov, Cosmological perturbations in SFT inspired nonlocal scalar field models, Eur. Phys. J. C 72 (2012) 2198, arXiv:0903.5176;

A.S. Koshelev and S.Yu. Vernov, Analysis of scalar perturbations in cosmological models with a non-local scalar field, Class. Quant. Grav. 28 (2011) 085019, arXiv:1009.0746;

N. Barnaby, A New Formulation of the Initial Value Problem for Nonlocal Theories, Nucl. Phys. B 845 (2011) 1, arXiv:1005.2945;

A. S. Koshelev, K. Sravan Kumar and P. Vargas Moniz, Effective models of inflation 
from a nonlocal framework, Phys. Rev. D 96 (2017) 103503

[20] S. Deser and R. P. Woodard, Nonlocal Cosmology, Phys. Rev. Lett. 99 (2007) 111301, arXiv:0706.2151.

[21] N. C. Tsamis and R. P. Woodard, A Caveat on Building Nonlocal Models of Cosmology, J. Cosmol. Astropart. Phys. 1409 (2014) 008 arXiv:1405.4470 [astro-ph.CO]].

[22] A. O. Barvinsky, Aspects of Nonlocality in Quantum Field Theory, Quantum Gravity and Cosmology, Mod. Phys. Lett. A 30 (2015) no.03n04, 1540003 arXiv:1408.6112 [hep-th]].

[23] S. Jhingan, S. Nojiri, S.D. Odintsov, M. Sami, I. Thongkool, and S. Zerbini, Phantom and non-phantom dark energy: The cosmological relevance of non-locally corrected gravity, Phys. Lett. B 663 (2008) 424, arXiv:0803.2613.

[24] J. Kluson, Non-Local Gravity from Hamiltonian Point of View, J. High Energy Phys. 1109 (2011) 001, arXiv:1105.6056.

[25] C. Deffayet and R.P. Woodard, Reconstructing the Distortion Function for Nonlocal Cosmology, J. Cosmol. Astropart. Phys. 0908 (2009) 023, arXiv:0904.0961.

[26] S. Deser and R. P. Woodard, Observational Viability and Stability of Nonlocal Cosmology, J. Cosmol. Astropart. Phys. 1311 (2013) 036 arXiv:1307.6639 [astroph.CO]].

[27] R. P. Woodard, Nonlocal Models of Cosmic Acceleration, Found. Phys. 44 (2014) 213 arXiv:1401.0254 [astro-ph.CO]].

[28] S. Nojiri and S.D. Odintsov, Modified non-local-F(R) gravity as the key for the inflation and dark energy, Phys. Lett. B 659 (2008) 821, arXiv:0708.0924].

[29] S. Park, Revival of the Deser-Woodard nonlocal gravity model: Comparison of the original nonlocal form and a localized formulation, Phys. Rev. D 97 (2018) 044006 arXiv:1711.08759 [gr-qc]].

[30] Y. l. Zhang, K. Koyama, M. Sasaki and G. B. Zhao, Acausality in Nonlocal Gravity Theory, J. High Energy Phys. 1603 (2016) 039 arXiv:1601.03808 [hep-th]].

[31] T.S. Koivisto, Dynamics of Nonlocal Cosmology, Phys. Rev. D 77 (2008) 123513, arXiv:0803.3399;

T.S. Koivisto, Newtonian limit of nonlocal cosmology, Phys. Rev. D 78 (2008) 123505, arXiv:0807.3778.

[32] S. Park and S. Dodelson, Structure formation in a nonlocally modified gravity model, Phys. Rev. D 87 (2013) 024003, arXiv:1209.0836.

[33] H. Nersisyan, A. F. Cid and L. Amendola, Structure formation in the Deser-Woodard nonlocal gravity model: a reappraisal, J. Cosmol. Astropart. Phys. 1704 (2017) no.04, 046 arXiv:1701.00434 [astro-ph.CO]].

[34] S. Nojiri, S.D. Odintsov, M. Sasaki and Y.l. Zhang, Screening of cosmological constant in non-local gravity, Phys. Lett. B 696 (2011) 278, arXiv:1010.5375.

[35] K. Bamba, S. Nojiri, S.D. Odintsov, and M. Sasaki, Screening of cosmological constant for De Sitter Universe in non-local gravity, phantom-divide crossing and finite-time future singularities, General Relativity and Gravitation 44 (2012) 1321, arXiv:1104.2692.

[36] Y.l. Zhang and M. Sasaki, Screening of cosmological constant in non-local cosmology, Int. J. Mod. Phys. D 21 (2012) 1250006, arXiv:1108.2112.

[37] E. Elizalde, E.O. Pozdeeva, and S.Yu. Vernov, De Sitter Universe in Non-local Gravity, Phys. Rev. D 85 (2012) 044002, arXiv:1110.5806;

S.Yu. Vernov, Nonlocal Gravitational Models and Exact Solutions, Phys. Part. Nucl. 43 (2012) 694, arXiv:1202.1172;

E. Elizalde, E.O. Pozdeeva, and S.Yu. Vernov, Reconstruction Procedure in Nonlocal Models, Class. Quantum Grav. 30 (2013) 035002, arXiv:1209.5957; 
E. Elizalde, E. O. Pozdeeva, S. Yu. Vernov and Y. 1. Zhang, Cosmological Solutions of a Nonlocal Model with a Perfect Fluid, J. Cosmol. Astropart. Phys. 1307 (2013) 034 arXiv:1302.4330 [hep-th]].

[38] S. Bahamonde, S. Capozziello and K. F. Dialektopoulos, Constraining Generalized Non-local Cosmology from Noether Symmetries, Eur. Phys. J. C 77 (2017) no.11, 722 arXiv:1708.06310 [gr-qc]].

[39] K.A. Bronnikov and E. Elizalde, Spherical systems in models of nonlocally corrected gravity, Phys. Rev. D 81 (2010) 044032, arXiv:0910.3929.

[40] S. Foffa, M. Maggiore and E. Mitsou, Cosmological dynamics and dark energy from nonlocal infrared modifications of gravity, Int. J. Mod. Phys. A 29 (2014) 1450116 arXiv:1311.3435 [hep-th]].

[41] Y. Dirian, S. Foffa, N. Khosravi, M. Kunz and M. Maggiore, Cosmological perturbations and structure formation in nonlocal infrared modifications of general relativity, J. Cosmol. Astropart. Phys. 1406 (2014) 033 arXiv:1403.6068 [astro-ph.CO]].

[42] M. Maggiore, Nonlocal Infrared Modifications of Gravity. A Review, Fundam. Theor. Phys. 187 (2017) 221 arXiv:1606.08784 [hep-th]].

[43] Y. Dirian, S. Foffa, M. Kunz, M. Maggiore and V. Pettorino, Non-local gravity and comparison with observational datasets, J. Cosmol. Astropart. Phys. 1504 (2015) no.04, 044, arXiv:1411.7692 [astro-ph.CO]];

Y. Dirian, S. Foffa, M. Kunz, M. Maggiore and V. Pettorino, Non-local gravity and comparison with observational datasets. II. Updated results and Bayesian model comparison with $\Lambda$ CDM, J. Cosmol. Astropart. Phys. 1605 (2016) no.05, 068, arXiv:1602.03558 [astro-ph.CO]].

[44] E. Belgacem, Y. Dirian, S. Foffa and M. Maggiore, Nonlocal gravity. Conceptual aspects and cosmological predictions, J. Cosmol. Astropart. Phys. 1803 (2018) no.03, 002 arXiv:1712.07066 [hep-th]].

[45] S. Capozziello, E. Elizalde, S. Nojiri, and S.D. Odintsov, Accelerating cosmologies from non-local higher-derivative gravity, Phys. Lett. B 671 (2009) 193, arXiv:0809.1535

[46] A. S. Koshelev, Stable analytic bounce in non-local Einstein-Gauss-Bonnet cosmology, Class. Quant. Grav. 30 (2013) 155001 arXiv:1302.2140 [astro-ph.CO]].

[47] A. S. Koshelev, L. Modesto, L. Rachwal and A. A. Starobinsky, Occurrence of exact $R^{2}$ inflation in non-local UV-complete gravity, J. High Energy Phys. 1611 (2016) 067 arXiv:1604.03127 [hep-th]];

A. S. Koshelev, K. Sravan Kumar and A. A. Starobinsky, $R^{2}$ inflation to probe non-perturbative quantum gravity, J. High Energy Phys. 1803 (2018) 071 arXiv:1711.08864 [hep-th]]. 